\title{
The Inaction Effect in the Psychology of Regret
}

\author{
Marcel Zeelenberg \\ Tilburg University
}

\author{
Eric van Dijk \\ Leiden University
}

\author{
Kees van den Bos \\ Utrecht University \\ Rik Pieters \\ Tilburg University
}

\begin{abstract}
Previous research showed that decisions to act (i.e., actions) produce more regret than decisions not to act (i.e., inactions). This previous research focused on decisions made in isolation and ignored that decisions are often made in response to earlier outcomes. The authors show in 4 experiments that these prior outcomes may promote action and hence make inaction more abnormal. They manipulated information about a prior outcome. As hypothesized, when prior outcomes were positive or absent, people attributed more regret to action than to inaction. However, as predicted and counter to previous research, following negative prior outcomes, more regret was attributed to inaction, a finding that the authors label the inaction effect. Experiment 4, showing differential effects for regret and disappointment, demonstrates the need for emotion-specific predictions.
\end{abstract}

Sometimes when confronted with a bad decision outcome, people blame themselves and realize that if only they had acted differently, this outcome would have been better. This unpleasant feeling, experienced when people look back on bad decisions, is the emotion of regret. Regret is a common experience, well known to most, if not all, of us. A study of verbal expressions of emotions in everyday conversation revealed that regret was the second most frequently named emotion (only love was mentioned more frequently; Shimanoff, 1984). But regret is not only experienced often; it also has serious behavioral implications, stemming from both the anticipation and the experience of this emotion (for reviews, see Landman, 1993; Zeelenberg, 1999; Zeelenberg, Inman, \& Pieters, 2001). Given its frequent occurrence and its potentially strong implications for our day-to-day behavior, it is important to develop our insights into the psychology of regret.

One of the central issues in current regret research concerns the question of whether people regret the actions they have taken more than the actions they have foregone (i.e., inactions). Because of the large number of studies showing that outcomes achieved through action lead to more regret than do the same outcomes achieved through inaction (e.g., Baron \& Ritov, 1994; Connolly, Ordóñez, \& Coughlan, 1997; Gilovich \& Medvec, 1994, 1995; Gilovich, Medvec, \& Chen, 1995; Gleicher et al., 1990; Kahneman \& Tversky, 1982; Landman, 1987; Miller \& Taylor, 1995; N'gbala \& Branscombe, 1997; Ordóñez \& Connolly, 2000; Ritov \& Baron,

Marcel Zeelenberg, Department of Economic and Social Psychology, Tilburg University, Tilburg, the Netherlands; Kees van den Bos, Department of Social and Organizational Psychology, Utrecht University, Utrecht, the Netherlands; Eric van Dijk, Department of Social and Organizational Psychology, Leiden University, Leiden, the Netherlands; Rik Pieters, Department of Marketing, Tilburg University.

Correspondence concerning this article should be addressed to Marcel Zeelenberg, Department of Economic and Social Psychology, Tilburg University, P.O. Box 90153, 5000-LE Tilburg, the Netherlands. E-mail: m.zeelenberg@kub.nl
1995; Zeelenberg, Van der Pligt, \& Manstead, 1998; Zeelenberg, Van Dijk, \& Manstead, 1998, 2000), Gilovich and Medvec (1995) nominated this as "the clearest and most frequently replicated finding" (p. 380) in this domain. In the remainder of this article we refer to this phenomenon as the action effect. ${ }^{1}$

In the present article we try to enhance the understanding of the psychology of regret by focusing on the antecedents of the action effect. Previous regret research has largely ignored the fact that decisions are often made in response to outcomes, experiences, or events that occurred earlier. We find this unfortunate because the presence of these prior outcomes, experiences, or events may be very typical in everyday life and may have considerable impact on the regret experienced over current decision outcomes. In what follows, we reason that when prior outcomes are negative, people may feel inclined to take action to improve future outcomes, which may make action more normal than inaction. As a consequence of this hypothesized psychological process, people may regret inaction more than action, an effect that we label the inaction effect.

It is important to note the differences between our approach and that of Gilovich and Medvec (1994, 1995). Gilovich and Medvec also found instances in which inactions were regretted more than actions. However, these authors studied the temporal pattern of regret and showed the existence of an inaction effect for long-term regrets: When looking back, people experience most regret over the paths not taken. Hence, Gilovich and Medvec argued that, over time, a number of psychological processes decrease the regret of actions taken and bolster the regret of actions forgone. Kahneman

\footnotetext{
${ }^{1}$ This research finding has also been referred to as emotional amplification (Kahneman \& Miller, 1986), the actor effect (Landman, 1987), and omission bias (Ritov \& Baron, 1990). Note that not all research on action/inaction differences focuses on emotional reactions following outcomes. The term omission bias has also been used to refer to action/ inaction differences in morality and intention (e.g., Baron \& Ritov, 1994; Kordes-de Vaal, 1996; Ritov \& Baron, 1990, 1992, 1995; Spranca, Minsk, \& Baron, 1991).
} 
(1995) argued that the short-term and long-term regrets that Gilovich and Medvec investigated were actually two different types of regret: hot regret, which is the direct emotional reaction to the outcome, and wistful regret, which is the less intense emotion "associated with pleasantly sad fantasies of what might have been" (p. 391). In a recent publication, Gilovich, Medvec, and Kahneman (1998) agreed on the existence of hot and wistful regret, thereby restating the fact that, ceteris paribus, actions produce more hot regrets than do inactions. Our present studies show the contrary. That is, we show that in some situations inactions lead to more hot regret than do actions. In doing so, our research stays close to Kahneman and Tversky's (1982) original action effect research and its follow-ups. First, however, we have to introduce the action effect, because this effect is the starting point of our investigation.

\section{The Action Effect}

We can most easily illustrate the action effect by citing Kahneman and Tversky's (1982) first demonstration of it. These authors confronted participants with the following scenario:

Paul owns shares in company $A$. During the past year he considered switching to stock in company $B$, but he decided against it. He now finds out that he would have been better off by $\$ 1,200$ if he had switched to the stock of company $B$. George owned shares in company $B$. During the past year he switched to stock in company $A$. He now finds out that he would have been better off by $\$ 1,200$ if he had kept his stock in Company $B$. Who feels more regret? (p. 173)

A large majority of the participants indicated more regret for George, who acted, than for Paul, who decided not to act. Kahneman and Tversky (1982) explained their finding by arguing that actions result in more regret because inactions are more normal than action. That is, they argued that it is easier to undo the outcome by mentally mutating an abnormal cause (i.e., action) into a normal cause (i.e., inaction) than the reverse, and because affective reactions to outcomes are enhanced when actual outcomes can easily be imagined otherwise, actions result in stronger regret than inactions do.

With this example, Kahneman and Tversky (1982) convincingly illustrated their reasoning that the normality of an outcome has consequences for the affective reaction to that outcome. However, consequently, researchers may have overgeneralized the implications of this demonstration, interpreting it as if it shows that action always produces more regret than inaction. In contrast, we pursue and extend the original line of reasoning proposed by Kahneman and Tversky. That is, by showing the existence of an inaction effect, we aim to extend their ideas to situations in which prior outcomes are known. This is important because these situations are omnipresent and may resemble everyday life more than do situations in which there are no prior outcomes whatsoever. Moreover, by adopting a social-psychological perspective on the action effect, we aim to show that inactions may not always be more normal than actions but sometimes are more abnormal than actions.

Thus, a question we address in the present article is whether actions are as abnormal as is suggested by the robustness of the action effect (see also N'gbala \& Branscombe, 1997). We argue they are not. We reason that actions are only abnormal when there are no strong reasons to act, as is the case in situations studied thus far, such as in Kahneman and Tversky's (1982) original scenario.
Furthermore, we argue that when prior outcomes call for action, inaction is more abnormal than action.

\section{The Impact of Prior Outcomes}

The current interpretation of the action effect is that actions are often more abnormal than inactions are. As we have already noted, however, we feel that there may be many situations that clearly call for action. In fact, the supposition that actions are more abnormal than inactions seems to be contradicted by game-theoretical research showing that people often base their decisions on a simple win stay-lose change heuristic (e.g., Macy, 1995). Likewise, research in consumer psychology often shows that people only take action when a prior experience was negative. For example, in their study on brand switching, Tellis and Geath (1990) demonstrated that consumers primarily switch brands after a negative experience; after positive or neutral experiences consumers tend to remain inactive and stick to their chosen brand. Furthermore, indirect support for this reasoning comes from the colloquial expressions that one should never change a winning team and that if it ain't broke, don't fix it. These sayings suggest that one's decision to take action or not might be based on earlier outcomes. The sayings also imply that action should follow a negative prior outcome or event: Fix it when it is broken, and change a losing team.

Taken together, the above leads us to propose that negative prior outcomes can induce a tendency to act and, consequently, make action more normal than inaction. If so, this would imply that in the case in which negative prior outcomes demand action to be taken, an inaction effect should be found; that is, actions foregone are regretted more than actions taken. Thus, if after experiencing a negative outcome, one does not take action to prevent further losses, one would feel intense regret if these losses do occur (e.g., asking oneself, "Why didn't I do anything?"). However, if one did take action to prevent further losses but was unsuccessful, the regret will be less intense (e.g., saying to oneself, "At least I tried!").

It would be important for our understanding of the psychology of regret if we could provide supportive evidence for this line of reasoning. As we argue above, everyday decisions are rarely made in isolation. The above-mentioned examples also suggest that people's emotional reactions to the outcomes of these decisions may be influenced by prior outcomes, which might have implications for the action/inaction differences found in previous research. We are not aware of any research investigating the influence of prior outcomes on regret following current actions and inactions.

\section{Regret and Responsibility}

An important element in our reasoning is that people feel more responsible for abnormal outcomes and that responsibility for current outcomes is one of the primary causes of regret. According to regret theory (e.g., Bell, 1982; Loomes \& Sugden, 1982), regret is a counterfactual emotion that stems from a comparison between what is and what might have been. However, not every "might have been" is supposed to produce regret. Regret is assumed to originate from comparisons between a factual outcome and an outcome that might have been had one chosen another action. Because one could have prevented the occurrence of the negative 
outcome by choosing something different, regret is related to a sense of responsibility for the outcome. Sugden (1985) was among the first to make this link explicit. In his view, regret stems both from realizing that an alternative course of action would have been better and from blaming oneself for the original decision. Moreover, according to Sugden, regret arising from self-recrimination or self-blame is most pronounced when one's decision was unreasonable, inexplicable, or indefensible. This can be interpreted as another way of saying that regret is more extreme the more abnormal the decision was.

The role of responsibility in regret was supported in a recent set of experiments (see, Connolly et al., 1997; Ordóñez \& Connolly, 2000; Zeelenberg, Van Dijk, \& Manstead, 1998, 2000). In these experiments, using a scenario methodology similar to one used in action effect research, researchers manipulated responsibility to investigate its effects on regret. The conclusion of these experiments was that although regret in rare cases may be experienced in absence of responsibility, the typical regret experience clearly involves a feeling of responsibility. This is consistent with Frijda, Kuipers, and Ter Schure (1989), who found that regret is closely related to the appraisal of self-agency. This link between regret and responsibility may help to explain why Gilovich and Medvec (1994, Study 4), in a study of everyday regrets, found so few regrets concerning negative outcomes that had been imposed on people. These researchers asked people to recall their biggest regrets. Less than $5 \%$ of these regrets involved outcomes caused by circumstances beyond the regretter's control.

The action effect seems to be congruent with the postulated relation between responsibility and regret. Earlier research showed that people who cause harm by acting are judged to be more immoral and more personally responsible than are people who cause the same harm by not acting (Ritov \& Baron, 1990; Spranca, Minsk, \& Baron, 1991). It has also been shown that actions, compared with inactions, are more salient, are more often used to infer one's own and others' attitude, and are perceived to be more informative (Fazio, Sherman, \& Herr, 1982). Together, these research findings suggest that the relation between a decision maker and a decision outcome is stronger for actions than for inactions because of the salience of the decision maker as a causal agent in the case of action. As Kahneman and Miller (1986) put it, "The findings that acts of commission produce greater regret than acts of omission . . . is in accord with formulations that distinguish omission from commission in attributions of causality and responsibility" (p. 145).

In summary, we argue for a mediating role of responsibility in action and inaction effects, and we propose that people experience regret over negative outcomes for which they feel responsible. More specifically, we propose that people feel responsible for outcomes that stem from actions taken when there were no strong reasons to act. Hence, if these actions result in bad outcomes, regret is amplified. However, we also argue that negative prior outcomes can provide reasons for action, which in terms of norm theory (Kahneman \& Miller, 1986; Kahneman \& Tversky, 1982) could be interpreted as showing that actions can sometimes be more abnormal than inactions. In these cases, people feel more responsible for negative outcomes when they are produced by a failure to act than when they are produced by an action. Consequently, in these cases, peoples' regret following inaction is amplified.

\section{Overview of the Present Studies}

In this article we test these ideas in a series of experiments that include all the important features present in Kahneman and Tversky's (1982) original scenario: “The consequences [of action and inaction] are precisely known and precisely matched, the regretted outcome is recent, and the emotion may be fairly intense" (cf. Kahneman, 1995, p. 389). Furthermore, we investigate our predictions using scenarios from a domain that is appealing to our participant population, namely, soccer coach decisions. ${ }^{2}$

Experiment 1 investigates the occurrence of the inaction effect; that is, whether inaction promotes more regret than does action in the case of negative prior outcomes. The hypothesized mediating role of responsibility in action and inaction effect is tested for in Experiment 2. In Experiment 3, we explore the boundary conditions under which our analysis of the inaction effect does not hold. Although our main interest is to demonstrate the inaction effect in regret, it may be useful to investigate the action/inaction effect in related emotions, such as disappointment. Differential effects on different emotions call for a need to be specific in theoretical as well as measurement issues (cf. DeSteno, Petty, Wegener, \& Rucker, 2000; Lerner \& Keltner, 2001; Zeelenberg, Van Dijk, \& Manstead, 1998). Therefore, Experiment 4 investigates whether the inaction effect is specific for regret or also generalizes to other emotions, such as disappointment.

\section{Experiment 1: The Inaction Effect}

Experiment 1 tests whether an inaction effect occurs when prior outcomes were negative. To achieve this, we manipulated outcomes that occurred before the regretted decision by having participants read a scenario in which soccer coaches either won or lost a match prior to the current one. The experiment also included a control condition in which prior outcomes were absent and in which the findings of previous studies should be replicated (showing an action effect). In the prior-outcome-positive condition, an action effect should also be found. This may be seen as an instance of the never change a winning team heuristic. Hence, when in response to a positive prior outcome a decision maker decides to take action and this action produces a negative outcome, the regret should be especially painful. This may result in an even larger action effect. In contrast, we also predicted that the prior-outcomenegative condition would result in an inaction effect. After a negative outcome, one should try to prevent the same thing from happening again by taking some sort of action. The regret one feels when the action results in another negative outcome should be less severe than the regret one feels when such a repeated negative outcome stems from inaction.

\section{Method}

One hundred sixty-five students (of whom 54 were men and 111 were women) at Leiden University, Leiden, the Netherlands, participated voluntarily and were paid for their participation. They were randomly assigned to one of three conditions (prior outcome: positive, absent, or negative). All

\footnotetext{
${ }^{2}$ Soccer is by far the most popular sport in the Netherlands. Important games are broadcast nationwide on television and attract the attention of the majority of potential viewers.
} 
participants were presented with a scenario that described two coaches, Steenland and Straathof, each in charge of a different soccer team. Participants in the prior-outcome-negative condition read the following scenario:

Steenland and Straathof are both coach of a soccer team. Steenland is the coach of Blue-Black, and Straathof is the coach of E.D.O. Both coaches lost the prior game with a score of 4-0. This Sunday Steenland decides to do something: He fields three new players. Straathof decides not to change his team. This time both teams lose with 3-0. Who feels more regret, coach Steenland or coach Straathof?

In the prior-outcome-positive condition, participants learned that both teams had won their previous match with a score of 4-0. In the prioroutcome-absent condition, no information about prior outcomes was given.

\section{Results}

The results are presented in Table $1 .^{3}$ As a replication of earlier research, an action effect was found for the prior-outcome-absent condition, $\chi^{2}(1, N=55)=13.26, p<.05$. Participants indicated that Coach Steenland (action) would feel more regret than Coach Straathof (inaction) would. This effect was also present in the prior-outcome-positive condition, $\chi^{2}(1, N=55)=36.82, p<.05$, and a direct comparison of these conditions showed that the effect was even more pronounced than in the prior-outcome-absent condition, $\chi^{2}(1, N=110)=4.07, p<.05$. In the prior-outcomenegative condition, however, the effect was reversed. Participants reported that Coach Straathof (inaction) would feel more regret than would Coach Steenland (action), $\chi^{2}(1, N=55)=6.56, p<$ .05 . This condition differed significantly from the other two, $\chi^{2} \mathrm{~s}(1$, $N \mathrm{~s}=110)>19, p \mathrm{~s}<.05$.

\section{Discussion}

The results clearly show the action effect when prior outcomes were unknown or positive. However, as predicted, the action effect did not occur when prior outcomes were negative. In fact, in this case the pattern reversed: The regret data show evidence for an inaction effect. This corroborates our line of thought that prior negative outcomes may provide a reason to act and thus make actions more normal and inactions more abnormal. As a consequence, decisions not to act that are followed by a negative outcome result in more regret than do decisions to act that lead to identical outcomes.

\section{Experiment 2: On the Role of Responsibility}

As we mentioned in the introduction, a central element of our line of reasoning is that the action effect is related to perceived responsibility for the outcome. In Experiment 1, however, we did not assess perceptions of responsibility. This information about perceived responsibility would provide insight into the process that, as we assumed, underlies our findings. We developed Experiment 2 for this purpose.

In Experiment 2 we aim to make the following extensions. First, we included a measure of perceived responsibility to test whether the manipulation of action/inaction and prior outcome indeed influenced the responsibility for the outcome. Second, in addition to the regret questions used in Experiment 1 (the dichotomous question about who feels more regret, the active or passive actorsimilar to the question used by Kahneman and Tversky, 1982), we
Table 1

Participants Reporting More Regret for Action or Inaction (Experiments 1, 2, and 4)

\begin{tabular}{|c|c|c|c|}
\hline \multirow[b]{2}{*}{ Experiment and condition } & \multicolumn{3}{|c|}{ Prior outcome } \\
\hline & $\begin{array}{l}\text { Positive } \\
\text { (won) }\end{array}$ & Absent & $\begin{array}{c}\text { Negative } \\
\text { (lost) }\end{array}$ \\
\hline \multicolumn{4}{|l|}{ Experiment 1} \\
\hline Action (Coach Steenland) & 50 & 41 & 18 \\
\hline Inaction (Coach Straathof) & 5 & 14 & 37 \\
\hline \multicolumn{4}{|l|}{ Experiment 2} \\
\hline Action (Coach Steenland) & 24 & 19 & 4 \\
\hline Inaction (Coach Straathof) & 1 & 6 & 21 \\
\hline \multicolumn{4}{|l|}{ Experiment 4} \\
\hline Action (Coach Steenland) & 44 & 41 & 18 \\
\hline Inaction (Coach Straathof) & 6 & 9 & 32 \\
\hline \multicolumn{4}{|l|}{ Overall } \\
\hline \multicolumn{4}{|l|}{ Action (Coach Steenland) } \\
\hline$\%$ & 91 & 78 & 31 \\
\hline$n$ & 118 & 101 & 40 \\
\hline \multicolumn{4}{|l|}{ Inaction (Coach Straathof) } \\
\hline$\%$ & 9 & 22 & 69 \\
\hline$n$ & 12 & 29 & 90 \\
\hline
\end{tabular}

also asked participants to indicate on 7-point scales the intensity of regret for both the active and the passive actor. These two new questions enable us to investigate the relation between responsibility and regret using the Baron and Kenny (1986) approach. More specifically, we expected that the effects of action/inaction and prior outcomes on regret would be mediated by perceptions of responsibility.

\section{Method}

Seventy-five students (44 women, 31 men) at Leiden University participated voluntarily and were paid for their participation. The design, materials, and procedure were similar to those in Experiment 1. After reading the scenario, participants were asked the following questions in the order described here. They were first asked questions concerning perceived responsibility for the outcomes: "To what extent is Steenland [Straathof] responsible for the defeat?" Both questions could be answered on 7-point scales, with higher scores indicating more responsibility for the outcome $(1=$ very little, $7=$ very much $)$. Participants were also asked to indicate the level of regret for both actors by means of the following question: "How much would Steenland [Straathof] regret his decision?" These questions were to be answered on 7-point scales, with higher scores indicating more regret $(1=$ very little, $7=$ very mисh $)$. Finally, they were asked the dichotomous question "Who feels more regret (Steenland or Straathof)?"

\section{Results}

Responsibility. The responsibility data (see Table 2 ) were submitted to a 3 (prior outcome: positive vs. absent vs. negative) $\times 2$ (decision: action vs. inaction) analysis of variance (ANOVA), with decision as a within-subject factor. This analysis yielded only a

\footnotetext{
${ }^{3}$ To facilitate the comparison of the different experiments, Table 1 also reports the replications of the results of Experiment 1 in Experiments 2 and 4.
} 
Table 2

Mean Levels of Responsibility and Regret in Experiment 2

\begin{tabular}{|c|c|c|c|c|c|c|}
\hline \multirow[b]{3}{*}{ Variable and condition } & \multicolumn{6}{|c|}{ Prior outcome } \\
\hline & \multicolumn{2}{|c|}{$\begin{array}{l}\text { Positive } \\
\text { (won) }\end{array}$} & \multicolumn{2}{|c|}{ Absent } & \multicolumn{2}{|c|}{$\begin{array}{l}\text { Negative } \\
\text { (lost) }\end{array}$} \\
\hline & $M$ & $S D$ & $M$ & $S D$ & $M$ & $S D$ \\
\hline \multicolumn{7}{|l|}{ Responsibility } \\
\hline Action (Coach Steenland) & $4.6_{\mathrm{a}}$ & 1.4 & $4.0_{\mathrm{a}}$ & 1.4 & $3.2_{\mathrm{b}}$ & 1.3 \\
\hline Inaction (Coach Straathof) & $2.9_{\mathrm{b}}$ & 1.2 & $4.0_{\mathrm{a}}$ & 1.6 & $5.2_{\mathrm{a}}$ & 1.0 \\
\hline \multicolumn{7}{|l|}{ Regret } \\
\hline Action (Coach Steenland) & $5.2 \mathrm{a}$ & 1.2 & $4.9 \mathrm{a}$ & 1.1 & $2.9_{\mathrm{b}}$ & 1.3 \\
\hline Inaction (Coach Straathof) & $3.3_{\mathrm{b}}$ & 1.3 & $4.0_{\mathrm{a}}$ & 1.6 & $4.8_{\mathrm{a}}$ & 1.6 \\
\hline
\end{tabular}

Note. Entries are mean ratings measured on 7-point scales, with higher scores indicating more responsibility and more regret. Means within columns that do not share a common subscript differ at $p<.05$.

significant Prior Outcome $\times$ Decision interaction, $F(2,72)=$ $29.65, p<.05$.

Comparisions of the relevant means showed that prior-outcomepositive participants indicated that the active coach was judged as being more responsible than the passive coach, $F(1,72)=38.72$, $p<.05$. In the prior-outcome-absent condition, they were judged equally responsible, $F(1,72)<1$. As predicted, in the prioroutcome-negative condition, the active coach was judged to be less responsible than the inactive coach $F(1,72)=52.02, p<.05$. These results show that manipulations of decision and prior outcomes influenced perceived responsibility for the current outcomes.

Regret. Participants were asked to indicate the levels of regret for both the active and the passive coach. The results, presented in Table 2, show the same pattern as the responsibility data addressed above. A 3 (prior outcome: positive vs. absent vs. negative) $\times$ (decision: action vs. inaction) ANOVA, with decision as a withinsubject factor, revealed a significant main effect for prior outcome, $F(2,72)=3.20, p<.05$, and a Prior Outcome $\times$ Decision interaction effect, $F(2,72)=21.32, p<.05$. Comparisons of the relevant means showed that prior-outcome-positive participants indicated more regret for the active coach than for the passive coach, $F(1,72)=20.04, p<.05$ (the action effect). This pattern was also present for prior-outcome-absent participants, $F(1$, $72)=3.84, p=.054$. The inaction effect was found for prioroutcome-negative participants, who indicated that the passive coach would feel more regret than the active coach, $F(1$, 72) $=20.04, p<.05$.

Participants' answers to the dichotomous question concerning who felt most regret are presented in Table 1. These data should, of course, be interpreted with caution, as they were collected after the continuous regret measure. Nevertheless, the results are virtually identical to those obtained in Experiment 1 and to the continuous measure in the present experiment. The action effect was found in the prior-outcome-absent condition, $\chi^{2}(1, N=25)=6.7$, $p<.05$, and in the prior-outcome-positive condition, $\chi^{2}(1, N=$ $25)=21.16, p<.05$. A direct comparison showed that the action effect was slightly more pronounced in the prior-outcome-positive condition, $p<.05$ ( $N=50$, one-sided Fisher exact probability test). More interesting, however, is the fact that the predicted inaction effect was found in the prior-outcome-negative condition, $\chi^{2}(1, N=25)=11.56, p<.05$. In a direct comparison, the results differed significantly from those in the other two conditions, $\chi^{2} \mathrm{~s}(1$, $N \mathrm{~s}=50)>18.00, p \mathrm{~s}<.05$.

Relating responsibility to regret: Mediation analysis. A central argument of the present article is that the amplification of regret is due to differences in perceived responsibility. When people feel highly responsible for an outcome, this should result in amplified regret. We have argued that prior outcomes can influence whether people feel more responsible for outcomes caused by action or by inaction. In other words, effects on perceived responsibility mediate the effects of prior outcomes on the action effect and inaction effect. The data presented so far are consistent with this view, but we have not yet provided an adequate test of this idea.

To test for mediation, we estimated a series of regression models (cf. Baron \& Kenny, 1986). The predictor variable in the models was the prior outcome manipulation. We effect coded this variable as follows: If the prior outcome was negative its value was -1 , if it was absent its value was 0 , and if it was positive its value was 1 . In this way, the effect-coded predictor ranged from inducing action (after a negative prior outcome), through a neutral point (when a prior outcome was absent), to inducing inaction (after a positive prior outcome). ${ }^{4}$ The hypothesized mediator was the difference in responsibility for the active and inactive coach (a positive value indicating more responsibility for the active coach). The difference in regret for the active and inactive coach (a positive value indicating more regret for the active coach) served as the dependent variable. To examine the degree of mediation, we first regressed the mediator on the predictor variable (Model 1), then regressed the dependent variable on the predictor variable (Model 2), then regressed the dependent variable on the mediator (Model 3), and finally regressed the dependent variable on both the predictor variable and the mediator (Model 4). The degree to which the influence of prior outcome on regret is reduced when one accounts for the influence of responsibility expresses the degree of mediation by responsibility. In case of mediation, the influence of prior outcomes dropped significantly, and in case of full mediation, the effect of prior outcomes became nonsignificant.

The results of these regression models are depicted in Figure 1. The numerical values in this figure are standardized regression weights. Responsibility clearly mediated the influence of prior outcomes on regret. Specifically, (a) prior outcomes predicted responsibility, $F_{\text {Model } 1}(1,73)=59.95$, regression weight $=0.67$, $p<.05$; (b) prior outcomes predicted regret, $F_{\text {Model } 2}(1$, $73)=39.25$, regression weight $=0.59, p<.05$; (c) responsibility predicted regret, $F_{\text {Model }}{ }_{3}(1,73)=136.83$, regression weight $=0.81, p<.05$; and (d) prior outcomes had no predictive power when we controlled for responsibility, $F_{\text {Model } 4}(2$, $72)=68.80$, regression weight between parentheses $=-0.09, n s$. In further support of our predictions, a regression model in which prior opponent predicted regret could be improved significantly by adding responsibility, $F_{\text {Model } 4-{ }_{2}}(1,72)=64.31, p<.05$,

\footnotetext{
${ }^{4}$ Analyses in which only the prior-outcome-negative and the prioroutcome-positive conditions or the prior-outcome-negative and prioroutcome-absent conditions were included showed similar patterns of results.
} 


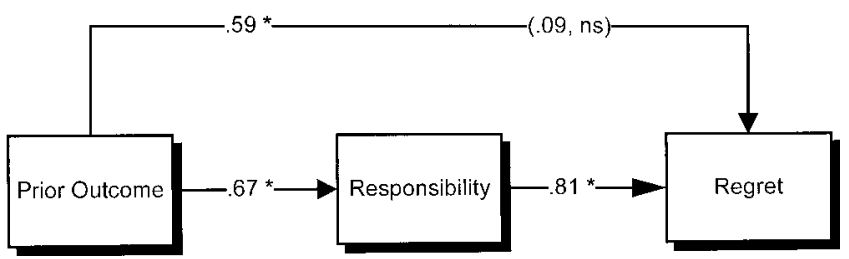

Figure 1. Testing the mediating role of responsibility on regret in Experiment 2. Data are standardized regression weights. ${ }^{*} p<.05$.

whereas a model in which responsibility predicted regret could not be improved by adding prior opponent, $F_{\text {Model } 4-3}(1,72)<1$. This clearly shows the mediation of responsibility between prior outcomes and regret.

\section{Discussion}

The aim of Experiment 2 was to investigate the effects of prior outcomes on regret following action and inaction and to relate these effects to perceived responsibility for that particular outcome. We found the action effect in the conditions in which prior outcomes were absent or positive. However, replicating the findings of Experiment 1, we found the inaction effect when prior outcomes were negative. These findings are in accordance with an explanation of the action effect in terms of perceptions of responsibility, as also shown by the reported mediation analysis.

\section{Experiment 3: Prior Outcomes Exert an Influence Only When They Are Informative}

We have argued that the inaction effect occurs because prior outcomes may call for action and that this is mostly the case when they are negative. In some instances, however, even negative prior outcomes may not be particularly informative. That is, sometimes prior outcomes do not signal a bad decision, and, hence, there is no need to take action. Then, action is not more normal than inaction. For example, when, in our soccer coach paradigm, an amateur soccer team has lost a game against a professional soccer team, there may be less need for the coach of the amateur team to question the strength of his team than after losing to another amateur team. Following this reasoning, the coach might be inclined to change the team after losing to another (essentially equally strong) amateur team but not after having lost from a much stronger professional team. Hence, the inaction effect-more regret after not having changed one's team-should occur after the team loses to an equally strong amateur team but should not occur after the team loses to a much stronger professional team. This hypothesis is examined in Experiment 3.

\section{Method}

One hundred students (48 men, 52 women) at Tilburg University, Tilburg, the Netherlands, participated voluntarily and were randomly assigned to one of the two conditions (professional opponent vs. amateur opponent). Participants in the professional opponent condition read the following scenario:

The soccer club "Vliestroom Boys" organizes a soccer tournament with six local amateur soccer teams and two professional soccer teams that rank high in the national Premier League (namely FeyenoordRotterdam and PSV-Eindhoven). Each team plays several games. Steenland and Straathof are both coach of an amateur team that participates in the tournament. Steenland is the coach of Blue-Black and Straathof is the coach of E.D.O. The teams of both coaches have played the previous game against one of the two professional teams.

Both the team of Steenland and the team of Straathof lost the previous game against the professional teams with a large margin.

In the next game, the teams of both coaches play against one of the other local amateur teams. Steenland, the coach of Blue-Black, decides to do something. He fields three different players. Straathof, the coach of E.D.O., decides not to change his team. Both teams lose this game.

The scenario in the amateur opponent condition was exactly the same, except that the opponents in the first game were two amateur teams instead of two professional teams. After reading the scenario, participants were asked to indicate "Who feels more responsible for this outcome?" and "Who experiences most regret for this outcome?" in both cases followed by response alternatives: Coach Steenland (who changed his team) or Coach Straathof (who left his team intact).

\section{Results}

The results are depicted in Table 3. This table is set up so that the results for regret and responsibility can be easily read out, as well as the relation between these two variables. Therefore, this table shows per condition (i.e., previous opponent: professional team vs. amateur team) a $2 \times 2$ table with the participants' responses to the questions "Who is more responsible?" and "Who feels more regret?" The four cells of each $2 \times 2$ table represent the four different combinations of the answers to the two questions. That is, when a participant answered that action would result in more responsibility and also in more regret, he or she ended up in the upper left cell of the $2 \times 2$ table. However, when he or she answered that action would result in more responsibility but inaction would result in more regret, he or she ended up in the lower left cell, and so on. The column totals of the $2 \times 2$ table represent the number of people who indicated action and inaction to the question "Who is more responsible?" and the row totals represent the answers to the question "Who feels more regret?" We first describe the findings for responsibility and regret that are shown in these row and column totals.

The results for responsibility were as expected. In the amateur opponent condition, the inactive coach was held more responsible for the defeat by 42 out of the 50 participants, $\chi^{2}(1, N=$ $50)=23.12, p<.05$, which replicates the inaction effect. In the professional opponent condition, the inactive and active coaches were equally often held most responsible for the defeat (22 participants indicated that action would be associated with more responsibility, whereas 28 participants indicated that inaction would be associated with more responsibility), $\chi^{2}(1, N=$ $50)=0.32, n s$. A direct comparison between the two conditions was also significant, $\chi^{2}(1, N=100)=9.33, p<.05$.

The results for regret show a pattern similar to that for responsibility. We expected to replicate the inaction effect in the amateur opponent condition, because there the prior defeat is meaningful, but not in the professional opponent condition, because there the prior defeat carries little information. The inaction effect was clearly replicated in the amateur opponent condition. The majority 
Table 3

Participants' Answers to the Questions Concerning Who Felt Most Regret and Responsibility for Both Previous Opponent Conditions (Experiment 3)

\begin{tabular}{|c|c|c|c|c|c|c|}
\hline \multirow[b]{4}{*}{ Who feels more regret? } & \multicolumn{6}{|c|}{ Previous opponent } \\
\hline & \multicolumn{3}{|c|}{ Amateur team } & \multicolumn{3}{|c|}{ Professional team } \\
\hline & \multicolumn{3}{|c|}{ Who is more responsible? } & \multicolumn{3}{|c|}{ Who is more responsible? } \\
\hline & Action & Inaction & Total & Action & Inaction & Total \\
\hline Action (Coach Steenland) & 3 & 9 & 12 & 18 & 5 & 23 \\
\hline Inaction (Coach Straathof) & 5 & 33 & 38 & 4 & 23 & 27 \\
\hline Total & 8 & 42 & & 22 & 28 & \\
\hline
\end{tabular}

Note. This table shows per condition (i.e., previous opponent: professional team vs. amateur team) a $2 \times 2$ table with the participants' responses to questions "Who is more responsible?" and "Who feels more regret?" Per condition, the column totals of the $2 \times 2$ table show the answers to the question "Who is more responsible?" and the row totals show the answers to the question "Who feels more regret?" (Thus, in the previous opponent amateur team condition, 8 participants indicated that action would result in more responsibility, whereas 42 participants indicated that inaction would result in more responsibility; and 12 participants indicated that action would result in more regret, whereas 38 participants indicated that inaction would result in more regret.) The entries in the $2 \times 2$ tables show how the participants' responses to the question concerning responsibility and regret are related. For example, the 3 in the upper left corner represents the 3 participants in the previous opponent amateur team condition who indicated that action (i.e., Coach Steenland, who took action) would result in more responsibility and more regret. The entry 5 (directly below the entry 3 ) represents the 5 participants in this condition who also indicated that action would result in more responsibility, while they indicated at the same time that inaction (i.e., Coach Straathof, who did not take action) would result in more regret.

of participants believed that inaction results in more regret $(38$ indicated inaction; 12 indicated action), $\chi^{2}(1, N=50)=13.52$, $p<.05$. As expected, no statistically significant difference between action and inaction was found in the professional opponent condition (23 participants indicated action; 27 indicated inaction), $\chi^{2}(1, N=50)=0.32, n s$. It is important to note that a direct comparison between the two conditions was also significant, $\chi^{2}(1$, $N=100)=5.32, p<.05$.

We now turn to the four cells of the $2 \times 2$ tables that are shown in Table 3. These cells show how the participants' responses to the questions concerning responsibility and regret are related. For example, the 3 in the upper left cell represents the 3 participants in the amateur team opponent condition who indicated that action (i.e., Coach Steenland, who took action) would result both in more responsibility and in more regret. The 5 in the lower left cell represents the 5 participants in this condition who also indicated that action would result in more responsibility; however, these participants indicated that inaction (i.e., Coach Straathof, who did not take action) would result in more regret. The fact that most observations are either in the upper left cell or in the lower right cell demonstrates the strong relation between regret and responsibility. That is, when more responsibility is attributed to inaction (or action), more regret is also attributed to inaction (or action). The precise relation between these two variables is addressed in the next section.

We performed mediation analysis similar to the one performed for Experiment 2. Because all variables were binary, we estimated a set of logit regression models (Long, 1997). The predictor variable in the models was the previous opponent: professional team (coded 0) or amateur team (coded 1). The hypothesized mediator was who was feeling more responsible for the defeat: the coach representing action (coded 0 ) or the coach representing inaction (coded 1). The dependent variable was who was experi- encing more regret: the coach representing action (coded 0 ) or the coach representing inaction (coded 1). First, the mediator was regressed on the predictor variable, next the dependent variable was regressed on the predictor variable, and finally the dependent variable was regressed on both the predictor variable and the mediator. The degree to which the influence of the prior opponent on regret was reduced when we accounted for the influence of responsibility expresses the degree of mediation by responsibility.

The results of these regression models are depicted in Figure 2. The numerical values in this figure and below are logit regression weights. Responsibility clearly mediates the influence of prior outcomes on regret. Specifically, (a) prior opponent predicted responsibility, $\chi_{\text {Model } 1}^{2}(1, N=100)=9.61$, regression weight $=1.42, p<.05$; (b) prior opponent predicted regret, $\chi_{\text {Model } 2}^{2}(1, N=100)=5.39$, regression weight $=0.99, p<.05$; (c) responsibility predicted regret, $\chi_{\text {Model } 3}^{2}(1, N=100)=22.78$, regression weight $=2.23, p<.05$; and (d) prior opponent had no predictive power when controlling for responsibility, $\chi_{\text {Model } 4}^{2}(1$, $N=100)=23.78$, regression weight between parentheses $=0.50$, $n s$. In further support of our predictions, a logit regression model in which prior opponent predicted regret could be improved sig-

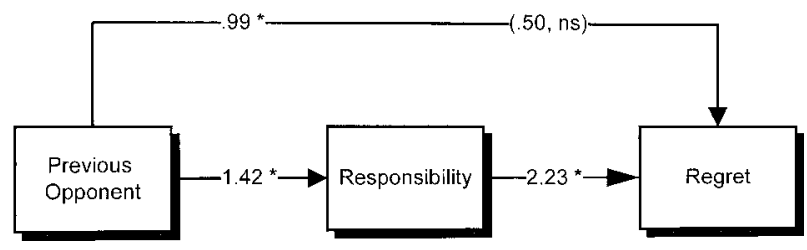

Figure 2. Testing the mediating role of responsibility on regret in Experiment 3: when prior outcomes are uninformative. Data are logit weights. ${ }^{*} p<.05$. 
nificantly by adding responsibility, $\chi_{\text {Model } 4-2}^{2}(1, N=100)=$ $18.39, p<.05$, whereas a model in which responsibility predicted regret could not be improved by adding prior opponent, $\chi_{\text {Model } 4-3}^{2}(1, N=100)=1.00, n s$. This again shows the mediation of responsibility between prior outcomes and regret.

\section{Discussion}

These results support our reasoning that if an amateur team loses to a professional team, the loss provides little information about the amateur team's ability, and hence there is no normal course of action (changing the team or not). But if an amateur team loses to another amateur team, this provides information that one should change the team to prevent future losses against other amateur teams. The results again strongly support that it is not action or inaction per se that determines the regret that people experience. Regret is an emotion that is functional in mastering skills and learning and in attaining a better grasp over decisions. It is especially salient in situations in which people should have known better, and not so much when this is not the case. Moreover, the impact of prior outcomes on regret is mediated by the responsibility for the decision.

\section{Experiment 4: Comparing Action/Inaction Effects for Regret and Disappointment}

In the previous experiments, we focused on effects of action/ inaction on regret. In the present experiment, we extend our focus and incorporate other emotional reactions to decision outcomes. We believe that this is an important experiment because in research on effects of action/inaction on emotional reactions, the dependent variable frequently is not regret but a more general and nonspecific measure of negative affect. For example, both Gleicher et al. (1990) and Zeelenberg, Van der Pligt, and De Vries (2000) confronted participants with action/inaction scenarios and measured general affective reactions. Gleicher et al. asked participants to indicate "who feels worse" (p. 289), and Zeelenberg et al. asked participants to indicate on an 11-point scale how bad (-5) or good (5) the active and the passive actor felt. More important, however, in studies directly aimed at regret, the measurement of the dependent variables has also been quite general. For example, Landman (1987, see p. 530) reported having asked participants to indicate "who feels worse." More recently, Connolly et al. (1997) investigated regret following action and inaction using an assessment of happiness with the obtained outcome on an 11-point scale ranging from -5 (very unhappy) to 5 (very happy).

We question whether these indirect measures teach us something about the psychology of regret. Put differently, when interested in regret, can we learn something by measuring related affective states? Recent research on the causes and consequences of specific emotions shows that one cannot simply assume that different emotions respond in the same way to identical manipulations, and, hence, this research stresses the need for emotionspecific theorizing (DeSteno et al., 2000; Lerner \& Keltner, 2001; Zeelenberg, Van Dijk, \& Manstead, 1998).

It seems worthwhile in this respect to differentiate between regret and disappointment, two important emotions people may experience after action/inaction decisions that go awry. Like regret, disappointment can be described as a feeling of negative affect. Indeed, on first sight, these emotions have a lot in common. They have both been called counterfactual emotions, both have negative valence, both are common experiences, and both are the result of decisions with unfortunate outcomes (Landman, 1993; Loomes \& Sugden, 1982, 1987; Roese, 1997; Roese \& Olson, 1997; Zeelenberg, Van Dijk, Manstead, \& Van der Pligt, 1998; Zeelenberg, Van Dijk, Manstead, \& Van der Pligt, 2000). On the basis of these similarities, one might be tempted to expect similar results for regret and disappointment.

However, we emphasize that regret and disappointment are not identical emotions and that the relation between prior outcomes and action/inaction effects may be different for both types of emotions. Previous research revealed that disappointment and regret do differ with regard to the experiential content (Zeelenberg, Van Dijk, Manstead, \& Van der Pligt, 1998), the way they are evoked (Van Dijk, Van der Pligt, \& Zeelenberg, 1999; Zeelenberg, Van Dijk, \& Manstead, 1998), and the different behavioral consequences these two emotions may produce (Zeelenberg \& Pieters, 1999). As Landman (1993) has argued, "an essential difference is disappointment's, and not regret's, dependence on expectations (estimated probabilities) of various outcomes" (p. 47; see also Van Dijk \& Van der Pligt, 1997). Experiments 1-3 of the current article inform us about the effects of manipulations of action/inaction and prior outcomes on regret. What can we expect for disappointment? Instead of predicting that these manipulations have similar effects on regret and disappointment, we predicted marked differences. Because disappointment results from outcomes that fall below expectations, it is important, we argue, to consider the effects of prior outcomes on expectations. We propose that manipulations of prior outcomes and action/inaction interact to affect expectations and, consequently, ratings of disappointment. For our soccer coach paradigm, we therefore predicted that participants in the prioroutcome-negative condition would report more disappointment after action than after inaction. After all, when a prior game was lost, expectations of winning the next game will not be high. Taking action to prevent further losses, however, may increase expectations, which causes disappointment following a loss to be more intense than it would be in the case of inaction. In a similar vein, we predicted expectations in the prior-outcome-positive condition to be influenced by the outcome of the prior game. After having won the prior game, expectations of winning the next game should be high. Taking action, changing the winning team, should decrease expectations. Losing the next game should then result in less disappointment compared with losing the game following inaction. Because the prior-outcome-absent condition did not contain information about the prior game on which expectations could be based, we had no a priori prediction about whether in this condition action or inaction would result in more disappointment.

Thus, the primary goal of this experiment, apart from replicating the main findings of Experiments 1-3, is to demonstrate that it is important to be specific about the emotion under investigation. As we have detailed above, we believe it is crucial to ask for specific ratings of that particular emotion, as action/inaction manipulations may result in completely opposite effects for different emotions.

\section{Method}

One hundred fifty students at Tilburg University (87 men, 63 women) participated voluntarily and were paid for their participation. They were 
randomly assigned to one of three conditions (prior outcome: positive vs. absent vs. negative). Participants first read the part of the scenario that introduced the two soccer teams and the results they obtained during the last game (the prior outcome), after which they read about the decisions of both soccer coaches for action and inaction. Then they were asked the following question to investigate the effects of action/inaction on their expectations: "Which coach has the highest expectation of winning the game of this Sunday?" After participants answered this question, they read on a subsequent page that both teams lost the Sunday game. Subsequently, they were asked, "Which coach feels more responsible for this outcome?" "Who feels more regret?" and "Who feels more disappointment?"

\section{Results and Discussion}

The results are depicted in Table 4. This table is set up similarly to Table 3, so that the results for regret and responsibility, and the results for disappointment and expectations, can be seen separately (in the row and column totals) as well as the relation between these variables (in the cells of the different $2 \times 2$ tables).

Responsibility and regret. The upper panel of Table 4 displays the results for regret and the hypothesized mediator responsibility. The results for responsibility were as expected. The active coach was judged as more responsible in the prior-outcome-absent condition (38 chose action vs. 12 chose inaction), $\chi^{2}(1, N=$ $50)=13.52, p<.05$, and in the prior-outcome-positive condition (48 chose action vs. 2 chose inaction), $\chi^{2}(1, N=50)=42.32, p<$ .05. A comparison of these two conditions showed that this effect was more pronounced in the latter condition, $\chi^{2}(1, N=$ $100)=8.31, p<.05$. It is important to note that in the prioroutcome-negative condition the effect was reversed, such that the inactive coach was judged to be more responsible than the active coach (18 chose action vs. 32 chose inaction), $\chi^{2}(1, N=$
$50)=3.92, p<.05$, which is consistent with the inaction effect. The results in this condition differed significantly from those obtained in the other two conditions, $\chi^{2} \mathrm{~s}(1, N \mathrm{~s}=100)>16, p \mathrm{~s}<$ .05 .

The results for regret mimic the findings of the earlier experiments (see also Table 1) and are in line with those for responsibility. The action effect was found in the prior-outcome-absent condition. Participants indicated that Coach Steenland (action) would feel more regret than would Coach Straathof (inaction; 41 chose action vs. 9 chose inaction), $\chi^{2}(1, N=50)=20.48, p<.05$. This effect was slightly more pronounced (but not significantly so) in the prior-outcome-positive condition (44 chose action vs. 6 chose inaction), $\chi^{2}(1, N=50)=28.88, p<.05$. In the prioroutcome-negative condition, however, the effect was reversed. Participants reported that the inactive coach would feel more regret than active coach (18 chose action vs. 32 chose inaction), $\chi^{2}(1$, $N=50)=3.92, p<.05$. This condition again differed significantly from the other two, $\chi^{2} \mathrm{~s}(1, N \mathrm{~s}=100)>21.00, p \mathrm{~s}<.05$.

Expectations and disappointment. The lower panel of Table 4 displays the results for disappointment and the hypothesized mediator expectations. The results for expectations are in line with our predictions. We reasoned that, overall, the expectations would be low in the prior-outcome-negative condition, as the teams lost the prior match. Changing the team (i.e., action) was expected to elevate expectations. Indeed, participants in this condition indicated that the expectations for the active coach would be higher (39 chose action vs. 11 chose inaction), $\chi^{2}(1, N=50)=15.68$, $p<.05$. We also reasoned that, overall, the expectations would be high following a prior victory (i.e., in the prior-outcome-positive condition) and that these would be decreased by taking action,

Table 4

Participants' Answers to the Questions Concerning Regret and Responsibility (Top Half) and Disappointment and Expectations (Bottom Half) for Each of the Three Prior Outcome Conditions (Experiment 4)

\begin{tabular}{|c|c|c|c|c|c|c|c|c|c|}
\hline \multirow[b]{4}{*}{ Who feels more regret? } & \multicolumn{9}{|c|}{ Prior outcome } \\
\hline & \multicolumn{3}{|c|}{ Positive } & \multicolumn{3}{|c|}{ Absent } & \multicolumn{3}{|c|}{ Negative } \\
\hline & \multicolumn{3}{|c|}{ Who is more responsible? } & \multicolumn{3}{|c|}{ Who is more responsible? } & \multicolumn{3}{|c|}{ Who is more responsible? } \\
\hline & Action & Inaction & Total & Action & Inaction & Total & Action & Inaction & Total \\
\hline Action (Coach Steenland) & 44 & 0 & 44 & 34 & 7 & 41 & 12 & 6 & 18 \\
\hline Inaction (Coach Straathof) & 4 & 2 & 6 & 4 & 5 & 9 & 6 & 26 & 32 \\
\hline \multirow[t]{2}{*}{ Total } & 48 & 2 & & 38 & 12 & & 18 & 32 & \\
\hline & \multicolumn{3}{|c|}{ Who had higher expectations? } & \multicolumn{3}{|c|}{ Who had higher expectations? } & \multicolumn{3}{|c|}{ Who had higher expectations? } \\
\hline Who feels more disappointment? & Action & Inaction & Total & Action & Inaction & Total & Action & Inaction & Total \\
\hline Action (Coach Steenland) & 7 & 13 & 20 & 15 & 14 & 29 & 31 & 6 & 37 \\
\hline Inaction (Coach Straathof) & 5 & 25 & 30 & 2 & 19 & 21 & 8 & 5 & 13 \\
\hline Total & 12 & 38 & & 17 & 33 & & 39 & 11 & \\
\hline
\end{tabular}

Note. This table shows per condition (i.e., prior outcome: positive vs. absent vs. negative) a $2 \times 2$ table within the top half the participants' responses to questions "Who is more responsible?" and "Who feels more regret?" and in the bottom half the participants' responses to questions "Who had higher expectations?" and "Who feels more disappointment?" In the top half, the column totals of each $2 \times 2$ table show the answers to the question "Who is more responsible?" and the row totals show the answers to the question "Who feels more regret?" The entries in the $2 \times 2$ tables in the top half show how the participants' responses to the question concerning responsibility and regret are related. In the bottom half, the column totals show the answer to the question "Who had higher expectations?" and the row totals show the answers to the question "Who feels more disappointment?" The entries in the $2 \times 2$ tables in the bottom half show how the participants' responses to the question concerning expectations and disappointment are related. 
resulting in lower expectations following action. This is what we found (12 chose action vs. 38 chose inaction), $\chi^{2}(1, N=$ $50)=13.52, p<.05$. We had no predictions for the prioroutcome-absent condition. The results show that participants indicated higher expectations following inaction (17 chose action vs. 33 chose inaction), $\chi^{2}(1, N=50)=5.12, p<.05$.

The results for disappointment were as expected and, hence, very different from the results for regret. In the prior-outcomepositive condition, participants indicated more disappointment following inaction, albeit not significantly so (20 chose action vs. 30 chose inaction), $\chi^{2}(1, N=50)=2.00, n s$. Participants in the prior-outcome-negative condition indicated more disappointment following action ( 37 chose action vs. 13 chose inaction), $\chi^{2}(1, N=$ $50)=11.52, p<.05$. Note that for these two conditions the effects are completely opposite to what was found for regret. Participants in the prior-outcome-absent condition indicated more disappointment following action than following inaction, but this difference was not significant (29 chose action vs. 21 chose inaction), $\chi^{2}(1$, $N=50)=1.28, n s$.

Relating responsibility to regret and expectations to disappointment: Mediation analysis. Next, we examined the mediation effect of responsibility on regret and of expectations on disappointment (see Figure 3), again by estimating sets of logit regression models (Long, 1997). The predictor variable in the models was the prior outcome manipulation. As in Experiment 2, this variable was effect coded: If the prior outcome was negative its value was -1 , if it was absent its value was 0 , and if it was positive its value was 1.

The results for regret are reported first (logit regression weights and $p$ values). As expected, responsibility mediated the influence of prior outcome on regret. Specifically, the analyses showed that (a) prior outcomes predicted responsibility, $\chi_{\text {Model 1 }}^{2}(1, N=$ 150) $=47.59$, regression weight $=1.82, p<.05$; (b) prior outcomes predicted regret, $\chi_{\text {Model } 2}^{2}(1, N=150)=34.03$, regression weight $=1.43, p<.05$; (c) responsibility predicted regret, $\chi_{\text {Model } 3}^{2}(1, N=150)=49.57$, regression weight $=2.79, p<.05$; (d) the influence of prior outcomes on regret was reduced by almost half when responsibility was accounted for, although it still remained significant, $\chi_{\text {Model } 4}^{2}(2, N=150)=56.78$, regression weight $=0.84, p<.05$. In further correspondence with these
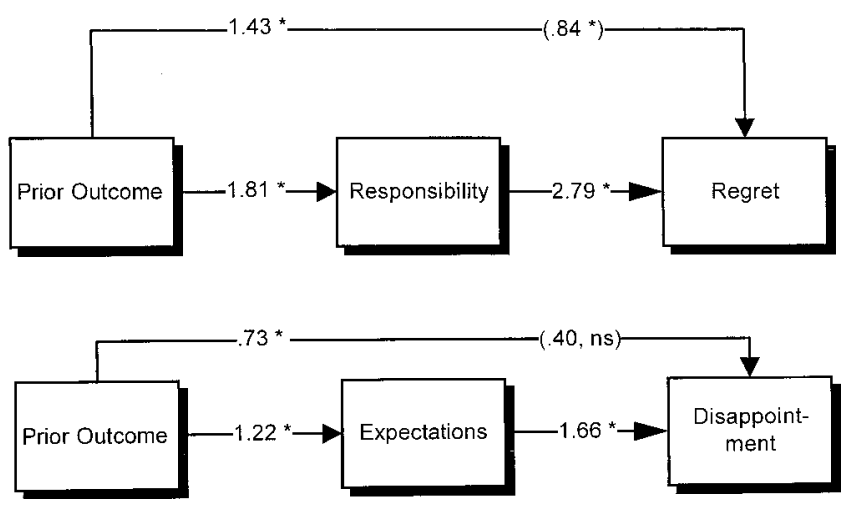

Figure 3. Testing the mediating role of responsibility on regret and expectations on disappointment in Experiment 4. Data are logit weights. ${ }^{*} p<.05$. findings, a logit regression model in which responsibility predicted regret could be improved by adding the prior outcome variable, $\chi_{\text {Model } 4-2}^{2}(1, N=150)=7.21, p<.05$, whereas a model in which the prior outcome variable predicted regret could be improved to a much larger extent by adding responsibility, $\chi_{\text {Model } 4-3}^{2}$ $(1, N=150)=22.75, p<.05$. This shows the, in this case partial, mediation of responsibility between prior outcomes and regret.

The results for disappointment are reported next. As we anticipated, expectations mediated the influence of prior outcome on disappointment. The results show that (a) prior outcomes predicted expectations, $\chi_{\text {Model } 1}^{2}(1, N=150)=31.09$, regression weight $=1.22, p<.05$; (b) prior outcomes predicted disappointment, $\chi_{\text {Model } 2}^{2}(1, N=150)=12.07$, regression weight $=0.73$, $p<.05$; (c) expectations predicted disappointment, $\chi_{\text {Model } 3}^{2}(1$, $N=150)=22.41$, regression weight $=1.66, p<.05$; and (d) the influence of prior outcomes on disappointment was insignificant when expectations were accounted for, $\chi_{\text {Model } 4}^{2}(2, N=150)=$ 25.17 , regression weight $=0.40, n s$. In further support of our predictions, a logit regression model in which the prior outcome variable predicted disappointment could be improved significantly by adding expectations, $\chi_{\text {Model } 4-2}^{2}(1, N=150)=13.01, p<$ .05 , whereas a model in which expectations predicted disappointment could not be improved by adding the prior outcome variable, $\chi_{\text {Model } 4-2}^{2}(1, N=150)=2.76, n s$. This shows the mediation of expectations between prior outcomes and disappointment.

The case for the influence of responsibility on regret (but not on disappointment) and for expectations on disappointment (but not on regret) was strengthened in two final logit regression analyses. In the first analysis, regret was predicted from the prior outcome variable and responsibility as well as from expectations. As expected, the responsibility variable was significant in this analysis, model- $\chi^{2}(3, N=150)=56.92$, regression weight $=2.24, p<.05$, but the expectations variable was not (regression weight $=0.19$, $p>.5)$. In the second analysis, disappointment was predicted from the prior outcome variable and expectations as well as from responsibility. As we anticipated, the expectations variable was significant in this analysis, model- $\chi^{2}(3, N=150)=25.21$, regression weight $=1.38, p<.05$, but the responsibility variable was not (regression weight $=-0.10, p>.5$ ).

This experiment reveals, as do the previous ones, that the effects of action/inaction on counterfactual emotions are very much contingent on what is known about prior outcomes. More important, the present experiment also shows that the effects of action/ inaction may be very different for regret and disappointment. This noteworthy finding corroborates both our view that it is important to be specific in theoretical and operational terms about the emotion under investigation and our claims about the restricted generalizability of the action effect.

\section{General Discussion}

The data presented in this article show that the action effect is not as robust as has been suggested by the overwhelming amount of replications reported in previous articles. The main difference between previous studies on the action effect and our current approach is that previous studies tended to concentrate on isolated decisions, whereas we studied sequences of decisions. From a theoretical standpoint, the notion of sequential decisions has generated new insights on the occurrence of action effects. In partic- 
ular, our results suggest that, depending on the valence of prior outcomes, inaction may result in more regret than action; that is, a reversal of "the clearest and most frequently replicated finding" (Gilovich \& Medvec, 1995, p. 380) in the regret domain. From a practical standpoint, one could argue that our current approach more closely resembles reality, in which decisions are rarely made in isolation. Instead, decisions usually follow on previous outcomes and decisions.

We regard it as telling that these findings are derived from experiments that, with the exception of the notion of prior outcomes, stay very close in methodology to the original work by Kahneman and Tversky (1982; cf. Kahneman, 1995). That is, participants were confronted with a scenario in which two persons arrive at the same negative outcome. One person arrives at this outcome after a decision to act, and the other person arrives at the same outcome through a decision not to act.

It is not only in terms of methodology that our current research builds on Kahneman and Tversky's (1982) pioneering work. Throughout the article, we relate our insights to the notion of normality in the sense that, like Kahneman and Tversky, we postulate that regret after abnormal decisions may be more extreme than after normal decisions. The innovative part of our approach is that we reason that normality of action and inaction may be dependent on prior outcomes. In the current article, we manipulated normality of actions and inactions by having participants read information about prior outcomes. This manipulation was inspired in part by research on the "win stay-lose change" heuristic and on consumer brand switching, which suggests that people mainly act when prior outcomes are negative (e.g., Macy, 1995; Tellis \& Geath, 1990). In the present experiments, the prior outcome was the outcome of the soccer game a team played the previous week. Consistent with predictions, we found that when the prior game was won or when there was no information about the prior game, participants reported that the coach who acted would feel more regret than would the coach who did not act. When the prior game was lost, participants indicated that the coach who acted would feel less regret than would the coach who did not act. The active coach at least tried to prevent further losses, whereas the inactive coach did nothing.

The finding that people regret negative outcomes that were caused by inactions more than they regret equally negative outcomes caused by actions when these outcomes were preceded by another negative outcome is consistent with an explanation of the action effect in terms of perceived responsibility. More support for this explanation was found in Experiments 2-4, in which analyses show mediation for perceived responsibility in the case of regret. Other findings from these experiments are also consistent with this reasoning.

It is appropriate to note that although we focus exclusively on the effects of prior outcomes, our point is actually much broader. ${ }^{5}$ Our manipulations of past outcomes are merely a vehicle to influence the normality of the actions that could produce regret. As Kahneman and Tversky (1982) and Kahneman and Miller (1986) argued, it is the normality of these behaviors that amplify the regret. We aim to show that in cases in which action is called for, unfortunate failures to act produce more regret. Action can be called for by negative prior outcomes, as we currently argue, but also by other factors, such as the social roles of some professions in which it is the person's job to act. Fire fighters, for example, are supposed to act in case of a fire and try to prevent bad things from happening. When they fail to act in these situations, they probably experience more regret and receive greater blame than do those who acted ineffectively. In other words, our current focus on the effects of prior outcomes is subordinate to the higher principle of normality.

\section{Scenario-Based Versus Real-Life Regrets}

The existence of an inaction effect and its interpretation in terms of perceived responsibility might also offer an explanation for Gilovich and Medvec's (1994, Study 5) failure to replicate the action/inaction finding for short-term real-life regrets. Gilovich and Medvec asked respondents to recall their single most regrettable action and inaction from the past week. Respondents indicated that they regretted recent actions as often as recent inactions. In a recent extension of this study, Feldman, Miyamoto, and Loftus (1999) asked participants about the intensity of both action and inaction regrets, and they also found that in real life these are equally intense. These findings thus contradict the earlier studies showing the action effect. Why is it that the most frequently replicated finding in scenario studies was not replicated in surveys of real-life regrets?

The answer might be related to the discrepancy between scenario-based and real-life regrets (see also Feldman et al., 1999). As we argue in this article, regret stems from the realization that an alternative course of action would have been better and from feelings of responsibility for the current outcome. Thus, regret arising from self-recrimination or self-blame is most pronounced when the decision was unreasonable, inexplicable, or indefensible (cf. Sugden, 1985). In the typical scenario study, participants learn about choices other people make and the outcomes associated with these actions without learning about the possible reasons why these people opt for action or inaction. In the Gilovich and Medvec (1994) and Feldman et al. (1999) surveys, respondents focused on their own real-life regrets. These respondents probably knew why they had chosen the regretted action or inaction and could probably explain these choices. Hence, there is no a priori reason to expect that the decisions not to act in these real-life samples were more explicable and defensible and thus associated with less responsibility than the decisions to act. This might explain why actions and inactions were equally regretted.

Thus, the findings of Gilovich and Medvec (1994) and of Feldman et al. (1999) can easily be accounted for in our reasoning. Of course, whether this explanation is valid cannot be determined on the basis of our data, and this is not the main focus of our investigation. Our main aim is to demonstrate the potential effects of prior outcomes on experienced regret and, more generally, to draw attention to the potential benefits of studying regret effects in a chain of events and decisions. By taking this into account and putting this notion at the heart of theorizing, one not only stays closer to the way decisions are embedded in reality but, as the current results indicate, one may also generate new and challenging findings.

\footnotetext{
${ }^{5}$ We thank an anonymous reviewer for stimulating us to address the more general implications of our theorizing.
} 
A second discrepancy between scenario-based and real-life regrets is the fact that participants in scenario studies do not actually experience regret but rather make predictions about what others, given a particular set of circumstances, might experience. Hence, given this projective nature of our findings, we have to be cautious in drawing conclusions about actual regret experiences and their behavioral implications. We have reasons to believe, however, that the current findings provide valuable insights into the psychology of regret. First, even when one has serious doubts about the correspondence between these imagined regrets and actual experienced regrets, one has to acknowledge that our data provide insights into what people anticipate regretting later on (cf. Connolly et al., 1997). These anticipated regrets have been shown to impact behavioral choice (for reviews, see Mellers, 2000; Zeelenberg, 1999). Second, many of the regret research findings, most of which are cited in this article, have been replicated using a whole range of methodologies, including scenario studies and recalled life experiences. In what follows, we assume sufficient correspondence between scenario-based regret and real-life regrets to speculate about the implications of our findings for the dynamics of regret.

\section{Regret in the Action Sequence}

Our findings thus suggest that it is worthwhile to shift attention to situations in which actions are not made in isolation but instead are embedded in an action sequence. When one thinks further along this line, the findings also draw attention to possible effects of experienced regret on subsequent behavior.

Although there is a large, emerging stream of research on the antecedents and consequences of anticipated regret in decision making, few studies have examined the more dynamic aspects of regret (cf. Zeelenberg et al., 2001). For example, what is the influence of the experience of regret on subsequent decisions, how can it moderate intention-choice relationships, and, more generally, what is regret's role in causing behavior? In a review of the role of feelings in the action sequence, Schwartz and Bohner (1996, p. 128) concluded that empirical research is mostly related to predecision and preaction processes such as determination of goal desirability and categorization of alternatives. Such research has emphasized the diagnostic function that global, environmentally induced moods such as happiness or sadness have in people's cognitive processes. Although emotion research has established that specific negative emotions such as regret, disappointment, fear, and anger are accompanied by very different thoughts, feelings, action tendencies, and goals (Frijda et al., 1989; Roseman, Wiest, \& Swartz, 1994), research on the role of specific emotions in the action and postaction phases is virtually absent.

Our present series of studies suggests that the specific emotion of regret is highly sensitive to prior outcomes and that these prior outcomes signal the need for action or inaction. Taking this perspective, one may argue that the emotion regret is informative with regard to the current status of individuals' goals (Lecci, Okun, \& Karoly, 1994) and may result in a focus on nonattained goals and, consequently, promote goal persistence. Thus, regret can be viewed as a goal-directed emotion (Bagozzi, Baumgartner, \& Pieters, 1998; Bagozzi, Baumgartner, Pieters, \& Zeelenberg, 2000), able to influence the expenditure of effort during the action phase. This implies that experiences of regret may strongly affect people's behaviors. In the future, researchers may want to examine more closely the role of regret experiences in goal striving and goal-directed behavior. For instance, it seems valuable to gain insight into the effects of regret on self-protective behaviors. There is evidence for beneficial effects of anticipated regret on selfprotection (Richard, Van der Pligt \& De Vries, 1996; Josephs, Larrick, Steele, \& Nisbett, 1992), but it is not yet clear whether and how the experience of the emotion itself, rather than its anticipation, may influence behaviors.

How does this relate to action and inaction regrets? It is interesting that research on inaction inertia (Tykocinski \& Pittman, 1998) suggests that regrets over inaction may reduce the likelihood that one acts on a subsequent opportunity that is still attractive but less attractive than the one missed earlier. However, we argue that the opposite may happen when the new opportunity is more attractive than the missed one. In that case, the regret experienced over the failure to act may promote action to be taken on the new opportunity. Similarly, action regrets may cause one to act more prudently in the future and, hence, their effect can be seen as inhibiting behavior, but research on consumer regrets has shown that the experience of regret promotes switching behavior (Tsiros \& Mittal, 2000; Zeelenberg \& Pieters, 1999; Zeelenberg et al., 2001). Future research should investigate the conditions under which action and inaction regrets are mobilizing or paralyzing.

However, to return to the concept that motivates the research we report here, compared with previous studies, the findings of the present article tell us something that is fundamental with regard to the psychology of regret. The present findings show that prior decision outcomes influence people's attributions of feelings of regret following action and inaction. What is especially interesting about the current findings is that they suggest that negative prior decision outcomes can promote action tendencies and, hence, may make people regret inaction more than action. In this way, the present article explores the inaction effect in the psychology of regret.

\section{References}

Bagozzi, R. P., Baumgartner, H., \& Pieters, R. (1998). Goal-directed emotions. Cognition and Emotion, 12, 1-26.

Bagozzi, R., Baumgartner, H., Pieters, R., \& Zeelenberg, M. (2000). The role of emotions in goal directed behavior. In S. Ratneshwar, D. G. Mick, C. Huffman (Eds.), The why of consumption (pp. 36-58). London: Routledge.

Baron, J., \& Ritov, I. (1994). Reference points and omission bias. Organizational Behavior and Human Decision Processes, 59, 475-498.

Baron, R. M., \& Kenny, D. A. (1986). The moderator-mediator variable distinction in social psychological research: Conceptual, strategic, and statistical considerations. Journal of Personality and Social Psychology, 51, 1173-1182.

Bell, D. E. (1982). Regret in decision making under uncertainty. Operations Research, 30, 961-981.

Connolly, T., Ordóñez, L. D., \& Coughlan, R. (1997). Regret and responsibility in the evaluation of decision outcomes. Organizational Behavior and Human Decision Processes, 70, 73-85.

DeSteno, D., Petty, R. E., Wegener, D. T., \& Rucker, D. D. (2000). Beyond valence in the perception of likelihood: The role of emotion specificity. Journal of Personality and Social Psychology, 78, 397-416. 
Fazio, R. H., Sherman, S. J., \& Herr, P. M. (1982). The feature-positive effect in the self-perception process: Does not doing matter as much as doing? Journal of Personality and Social Psychology, 42, 404-411.

Feldman, J., Miyamoto, J., \& Loftus, E. F. (1999). Are actions regretted more than inactions? Organizational Behavior and Human Decision Processes, 78, 232-255.

Frijda, N. H., Kuipers, P., \& Ter Schure, E. (1989). Relations among emotion, appraisal, and emotional action readiness. Journal of Personality and Social Psychology, 57, 212-228.

Gilovich, T., \& Medvec, V. H. (1994). The temporal pattern to the experience of regret. Journal of Personality and Social Psychology, 67, 357-365.

Gilovich, T., \& Medvec, V. H. (1995). The experience of regret: What, when, and why. Psychological Review, 102, 379-395.

Gilovich, T., Medvec, V. H., \& Chen, S. (1995). Omission, commission, and dissonance reduction: Overcoming regret in the Monty Hall problem. Personality and Social Psychology Bulletin, 21, 182-190.

Gilovich, T., Medvec, V. H., \& Kahneman, D. (1998). Varieties of regret: A debate and partial resolution. Psychological Review, 105, 602-605.

Gleicher, F., Kost, K. A., Baker, S. M., Strathman, A. J., Richman, S. A., \& Sherman, S. J. (1990). The role of counterfactual thinking in judgments of affect. Personality and Social Psychology Bulletin, 16, 284295.

Josephs, R. A., Larrick, R. P., Steele, C. M., \& Nisbett, R. E. (1992). Protecting the self from the negative consequences of risky decisions. Journal of Personality and Social Psychology, 62, 26-37.

Kahneman, D. (1995). Varieties of counterfactual thinking. In N. J. Roese \& J. M. Olson (Eds.), What might have been: The social psychology of counterfactual thinking (pp. 375-396). Mahwah, NJ: Erlbaum.

Kahneman, D., \& Miller, D. T. (1986). Norm theory: Comparing reality to its alternatives. Psychological Review, 93, 136-153.

Kahneman, D., \& Tversky, A. (1982). The psychology of preferences. Scientific American, 246, 160-173.

Kordes-de Vaal, J. (1996). Intention and the omission bias: Omissions perceived as nondecisions. Acta Psychologica, 93, 161-172.

Landman, J. (1987). Regret and elation following action and inaction: Affective reactions to positive versus negative outcomes. Personality and Social Psychology Bulletin, 13, 524-536.

Landman, J. (1993). Regret: The persistence of the possible. New York: Oxford University Press.

Lecci, L., Okun, M. A., \& Karoly, P. (1994). Life regrets and current goals as predictors of psychological adjustment. Journal of Personality and Social Psychology, 66, 731-741.

Lerner, J. S., \& Keltner, D. (2001). Fear, anger, and risk. Journal of Personality and Social Psychology, 81, 146-159.

Long, J. S. (1997). Regression models for categorical and limited dependent variables. Thousand Oaks, CA: Sage.

Loomes, G., \& Sugden, R. (1982). Regret theory: An alternative theory of rational choice under uncertainty. Economic Journal, 92, 805-824.

Loomes, G., \& Sugden, R. (1987). Testing for regret and disappointment in choice under uncertainty. Economic Journal, 97, 118-129.

Macy, M. W. (1995). PAVLOV and the evolution of cooperation: An experimental test. Social Psychology Quarterly, 58, 74-87.

Mellers, B. A. (2000). Choice and the relative pleasure of consequences. Psychological Bulletin, 126, 910-924.

Miller, D. T., \& Taylor, B. R. (1995). Counterfactual thinking, regret, and superstition: How to avoid kicking yourself. In N. J. Roese \& J. M. Olson (Eds.), What might have been: The social psychology of counterfactual thinking (pp. 305-331). Hillsdale, NJ: Erlbaum.

N'gbala, A., \& Branscombe, N. R. (1997). When does action elicit more regret than inaction and is counterfactual thinking the mediator of this effect? Journal of Experimental Social Psychology, 33, 324-343.

Ordóñez, L. D., \& Connolly, T. (2000). Regret and responsibility: A reply to Zeelenberg et al. (1998). Organizational Behavior and Human Decision Processes, 81, 132-142.

Richard, R., Van der Pligt, J., \& De Vries, N. K. (1996). Anticipated affect and behavioral choice. Basic and Applied Social Psychology, 18, 111129.

Ritov, I., \& Baron, J. (1990). Reluctance to vaccinate: Omission bias and ambiguity. Journal of Behavioral Decision Making, 3, 263-277.

Ritov, I., \& Baron, J. (1992). Status quo and omission biases. Journal of Risk and Uncertainty, 5, 49-61.

Ritov, I., \& Baron, J. (1995). Outcome knowledge, regret and omission bias. Organizational Behavior and Human Decision Processes, 64, 119-127.

Roese, N. J. (1997). Counterfactual thinking. Psychological Bulletin, 121, $133-148$.

Roese, N. J., \& Olson, J. M. (1997). Counterfactual thinking: The intersection of affect and function. In M. P. Zanna (Ed.), Advances in experimental social psychology (Vol. 29, pp. 1-59). San Diego, CA: Academic Press.

Roseman, I. J., Wiest, C., \& Swartz, T. S. (1994). Phenomenology, behaviors, and goals differentiate discrete emotions. Journal of Personality and Social Psychology, 67, 206-211.

Schwartz, N., \& Bohner, G. (1996). Feelings and their motivational implications. In P. M. Gollwitzer \& J. A. Bargh (Eds.), The psychology of action: Linking cognition and motivation to behavior (pp. 119-145) New York: Guilford Press.

Shimanoff, S. B. (1984). Commonly named emotions in everyday conversations. Perceptual and Motor Skills, 58, 514.

Spranca, M., Minsk, E., \& Baron, J. (1991). Omission and commission in judgment and choice. Journal of Experimental Social Psychology, 27, 76-105.

Sugden, R. (1985). Regret, recrimination and rationality. Theory and Decision, 19, 77-99.

Tellis, G. J., \& Geath, G. J. (1990). Best value, price seeking, and price aversion: The impact of information and learning on consumer choices. Journal of Marketing, 54, 34-45.

Tsiros, M., \& Mittal, V. (2000). Regret: A model of its antecedents and consequences in consumer decision making. Journal of Consumer Research, 26, 401-417.

Tykocinski, O. E., \& Pittman, T. S. (1998). The consequences of doing nothing: Inaction inertia as avoidance of anticipated counterfactual regret. Journal of Personality and Social Psychology, 75, 607-616.

Van Dijk, W. W., \& Van der Pligt, J. (1997). The impact of probability and magnitude of outcome on disappointment and elation. Organizational Behavior and Human Decision Processes, 69, 277-284.

Van Dijk, W. W., Van der Pligt, J., \& Zeelenberg, M. (1999). Effort invested in vain: The impact of effort on the intensity of disappointment and regret. Motivation and Emotion, 23, 203-220.

Zeelenberg, M. (1999). Anticipated regret, expected feedback and behavioral decision-making. Journal of Behavioral Decision Making, 12, 93-106.

Zeelenberg, M., Inman, J. J., \& Pieters, R. G. M. (2001). What we do when decisions go awry: Behavioral consequences of experienced regret. In E. U. Weber, J. Baron, \& G. Loomes (Eds.), Conflict and tradeoffs in decision making (pp. 136-155). New York: Cambridge University Press.

Zeelenberg, M., \& Pieters, R. (1999). Comparing service delivery to what might have been: Behavioral responses to regret and disappointment. Journal of Service Research, 2, 86-97.

Zeelenberg, M., Van der Pligt, J., \& De Vries, N. K. (2000). Attributions of responsibility and affective reactions to decision outcomes. Acta Psychologica, 104, 303-315.

Zeelenberg, M., Van der Pligt, J., \& Manstead, A. S. R. (1998). Undoing regret on Dutch television: Apologizing for interpersonal regrets involv- 
ing actions or inactions. Personality and Social Psychology Bulletin, 24, $1114-1120$

Zeelenberg, M., Van Dijk, W. W., \& Manstead, A. S. R. (1998). Reconsidering the relation between regret and responsibility. Organizational Behavior and Human Decision Processes, 74, 254-272.

Zeelenberg, M., Van Dijk, W. W., \& Manstead, A. S. R. (2000). Regret and responsibility resolved? Evaluating Ordóñez and Connolly's (2000) conclusions. Organizational Behavior and Human Decision Processes, 81, 143-154.

Zeelenberg, M., Van Dijk, W. W., Manstead, A. S. R., \& Van der Pligt, J.
(1998). The experience of regret and disappointment. Cognition and Emotion, 12, 221-230.

Zeelenberg, M., Van Dijk, W. W., Manstead, A. S. R., \& Van der Pligt, J. (2000). On bad decisions and disconfirmed expectancies: The psychology of regret and disappointment. Cognition and Emotion, 14, 521-541.

Received June 7, 2000

Revision received July 23, 2001

Accepted July 26, 2001

\section{American Psychological Association SubSCRIPTION ClaIMS INFORMATION}

Today's Date:

We provide this form to assist members, institutions, and nonmember individuals with any subscription problems. With the appropriate information we can begin a resolution. If you use the services of an agent, please do NOT duplicate claims through them and directly to us. PLEASE PRINT CLEARLY AND IN INK IF POSSIBLE.

\begin{tabular}{llll}
\hline PRINT FULL NAME OR KEY NAME OF INSTITUTION & & \\
\hline ADDRESS & & & \\
\hline CITY & STATE/COUNTRY & ZIP
\end{tabular}

YOUR NAME AND PHONE NUMBER

TITLE

$$
\begin{aligned}
& \text { MEMBER OR CUSTOMER NUMBER (MAY BE FOUND ON ANY PAST ISSUE LABEL) } \\
& \hline \text { DATE YOUR ORDER WAS MARED (OR PHONED) } \\
& \text { (If possible, send a copy, front and back, of your cancelled check to help us in our research } \\
& \text { of your claim.) } \\
& \text { ISSUES:___ MISSING _ _ DAMAGED }
\end{aligned}
$$

VOLUME OR YEAR NUMBER OR MONTH

Thank you. Once a claim is received and resolved, delivery of replacement issues routinely takes 4-6 weeks.

(TO BE FILLED OUT BY APA STAFF)

DATE RECEIVED:

ACTION TAKEN:

STAFF NAME:
DATE OF ACTION: INV. NO. \& DATE: LABEL NO. \& DATE: 